\title{
Colección de Mamíferos, Museo de Historia Natural de la Universidad de Caldas (MHN-UCa)
}

\author{
Héctor E. Ramírez-Chaves ${ }^{1 *}$ (iD \\ 1 Departamento de Ciencias Biológicas, Facultad de Ciencias Exactas y Naturales, Universidad de Caldas, y Centro de Museos, \\ Museo de Historia Natural, Universidad de Caldas. Calle 65 \# 26-10, 170004, Manizales, Caldas, Colombia \\ * Correspondencia: hector.ramirez@ucaldas.edu.co
}

\section{Resumen}

La Colección de Mamíferos del Museo de Historia Natural de la Universidad de Caldas (MHN-UCa) alberga 3287 especímenes de mamíferos pertenecientes a 12 órdenes, 41 familias (38 nativas y 3 exóticas), 134 géneros (128 nativos y 6 exóticos) y 228 especies (7 exóticas). Los especímenes proceden de 23 departamentos del país. La colección es la más antigua y grande de este tipo en el Eje Cafetero y ha sido empleada en al menos 30 publicaciones científicas.

Palabras clave: Chiroptera, Eje Cafetero, especímenes, Manizales, Rodentia.

\begin{abstract}
The Mammals Collection of the Museo de Historia Natural de la Universidad de Caldas (MHN-UCa) holds 3287 specimens belonging to 12 orders, 41 families (38 native and 3 exotic), 134 genera (128 native and 6 exotic), and 228 species (7 exotic). The specimens come from 23 departments in the country. The collection is the oldest and largest of its kind in the Colombia Coffee region, and it has been used in at least 30 scientific publications.
\end{abstract}

Key words: Chiroptera, Coffee region, Manizales, specimens, Rodentia.

La Colección de Mamíferos del Museo de Historia Natural de la Universidad de Caldas (MHN-UCa) (Figura 1), está ubicada en Manizales - Caldas y fue fundada en 1976. En la actualidad, la colección alberga 3287 especímenes de mamíferos (conteo a 20 abril de 2020), pertenecientes a 12 órdenes, 41 familias (38 nativas y 3 exóticas: Cercopithecidae, Muridae y Suidae), 134 géneros (128 nativos y 6 exóticos: Felis, Macaca, Mesocricetus, Mus, Rattus, Sus) y 228 especies (7 exóticas; Tabla 1). En total, 3287 especímenes se encuentran catalogados y 10 están en proceso de ingreso a la colección (material en proceso de catalogación). Los órdenes mejor representados son Chiroptera (2210 especímenes) y Rodentia (583 especimenes) con 112 y 46 especies respectivamente (Tabla 1). 64 especimenes, principalmente de Rodentia, no han sido identificados a género. Los especimenes proceden de los departamentos de: Amazonas (3), Antioquia (102), Arauca (583), Bolívar (6), Boyacá (2), Caldas (1916), Casanare (6), Cauca (29), Cesar (4), Chocó (3), Cundinamarca (14), Huila (4), La Guajira (73), Magdalena (4), Meta (11), Nariño (59), Putumayo (8), Quindío (98), Risaralda (139), Santander (57), Tolima (125), Valle del Cauca (21) y Vichada (8). 12 especimenes no cuentan con localidad precisa. 


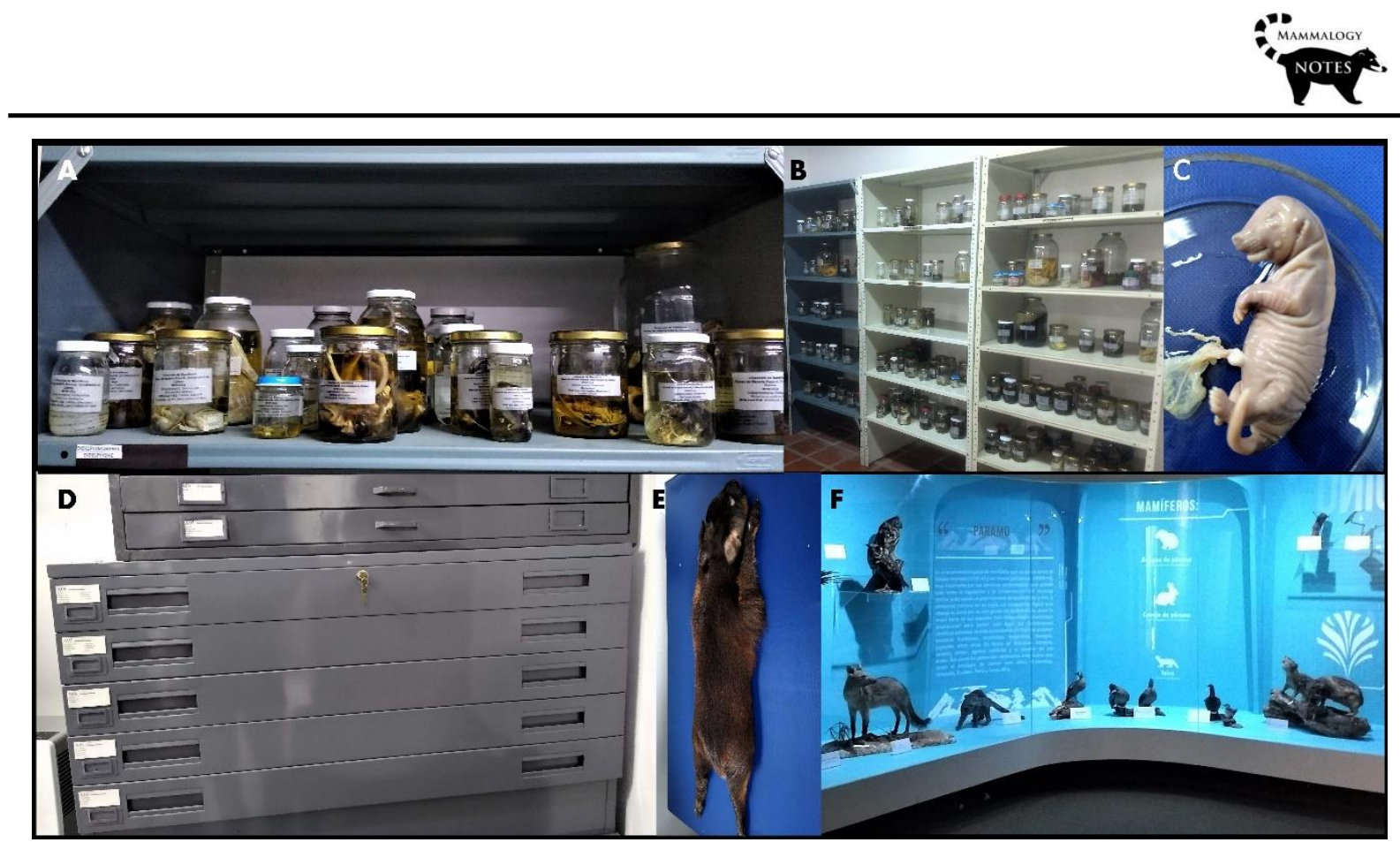

FIGURA 1. Detalles de la colección de Mamíferos del Museo de Historia Natural de la Universidad de Caldas (MHN-UCa). A-B: Detalles de la colección en líquido. C: Embrión de Conepatus semistriatus (MHN-UCa 3245). D: Detalles de los almacenadores de la colección en seco. E: Ejemplar de Mazama rufina (MHN-UCa 2946). F. Sección de exhibición del Centro de Museos, Museo de Historia Natural de la Universidad de Caldas.

Ejemplares de la colección de mamíferos del MHN-UCa, han sido empleados en al menos 30 publicaciones científicas (Sánchez 2000; Castaño et al. 2003a,b, 2004a,b, 2017; Sánchez \& Alvear 2003; Castaño \& Corrales 2004, 2010; Sánchez et al. 2004; Roncancio-D. \& EstévezV. 2007; Roncancio \& Ramírez-Chaves 2008; Castaño Salazar 2012; Marín et al. 2012; RamírezChaves et al. 2012, 2018; Escobar-Lasso \& Guzmán-Hernández 2014; Escobar-Lasso \& GilFernández 2014; Escobar-Lasso et al. 2013, 2014; Cardona et al. 2016; Díaz-Nieto \& Voss 2016; Castaño Ramírez \& Ramírez-Chaves 2018; García Restrepo et al. 2018; Marín-C. et al. 2018; Montilla et al. 2018; Serna-Botero et al. 2019; Torres-Martínez et al. 2019; Esquivel et al. 2020; Morales-Martínez et al. en prensa). El número de citaciones de los artículos que incluyen especímenes de la colección de Mamíferos del MHN-UCa, se encuentra disponible en el siguiente enlace: https: / cutt.ly/ neivflB

La colección cuenta con información de 2114 especímenes disponible en el siguiente enlace: https://www.gbif.org/dataset/1a58ec89-e5d4-4b2b-9ed3-1c8b5f70f4fc (RamírezChaves et al. 2019, 2020).

Entre los investigadores que aportaron al crecimiento de la colección de Mamíferos del MHN-UCa, se encuentran John Harold Castaño quien fortaleció la colección durante su paso como curador, al ingresar más de 1000 especímenes, formar varios mastozoólogos locales y contribuir, de manera considerable, al conocimiento de los mamíferos del Eje Cafetero mediante la divulgación de sus resultados de investigación. 
Mamíferos del Museo de Historia Natural de la Universidad de Caldas (MHN-UCa). Dos ejemplares no han sido aún identificados por tratarse de fragmentos óseos.

\begin{tabular}{lcccc}
\hline Orden & Familias & Géneros & Especies & Número de especímenes \\
\hline Didelphimorphia & 1 & 8 & 17 & 141 \\
Paucituberculata & 1 & 1 & 1 & 7 \\
Cingulata & 2 & 2 & 4 & 27 \\
Pilosa & 3 & 4 & 4 & 28 \\
Sirenia & - & - & - & - \\
Eulipotyphla & 1 & 1 & 3 & 69 \\
Chiroptera & 7 & 51 & 112 & 2210 \\
Carnivora & 6 & 18 & 20 & 120 \\
Perissodactyla & 1 & 1 & 2 & 2 \\
Artiodactyla & 3 & 5 & 7 & 30 \\
Cetacea & - & - & - & - \\
Primates & 5 & 9 & 10 & 28 \\
Rodentia & 10 & 33 & 46 & 583 \\
Lagomorpha & 1 & 1 & 2 & 40 \\
Total & 41 & 134 & 228 & 3285 \\
\hline
\end{tabular}

\section{AGRADECIMIENTOS}

A los Semilleros de Investigación en Mastozoología (SIMas), Primates (SIPCE) y Carnívoros (SIC) de la Universidad de Caldas, por su apoyo. A los integrantes del "Natural History Laboratory: Integrative Zoological Biodiversity Discovery" y al grupo GEBIOME por el apoyo en diferentes actividades de la colección.

\section{REFERENCIAS}

Cardona DM, Castaño JH, Botero JE. 2016. Distribución anidada de murciélagos en fragmentos de bosque premontano de la cuenca media del río Cauca, Colombia. Mastozoología Neotropical 23:371-387.

Castaño JH, Muñoz-Saba Y, Botero JE, Vélez JH. 2003a. Mamíferos del Departamento de Caldas Colombia. Biota Colombiana 4:247-259.

Castaño JH, Corrales JD, Velásquez S. 2003b. Estructura y composición de la comunidad de murciélagos de un fragmento de bosque andino en la ciudad de Manizales, Caldas. Boletín Científico, Centro de Museos, Museo de Historia Natural 7:113-120

Castaño JH, Corrales JD. 2004. Primer registro de Centronycteris centralis (Chiroptera: Emballonuridae) en los Andes colombianos. Mastozoología Neotropical 14:69-72.

Castaño JH, Botero JE, Velásquez S, Corrales JD. 2004a. Murciélagos en agroecosistemas cafeteros de Colombia. Chiroptera Neotropical 10:196-199. 
Castaño JH, Orrego Santa OA, Cardona Ramírez DM. 2004b. Primer registro para Caldas del Murciélago de Ventosas de Vientre Blanco (Thyroptera tricolor Spix, 1823). Boletín Científico, Centro de Museos, Museo de Historia Natural 8:260-266.

Castaño JH, Corrales JD. 2010. Mamíferos de la cuenca del río La Miel (Caldas): diversidad y uso cultural. Boletín Científico, Centro de Museos, Museo de Historia Natural 14:56-75.

Castaño JH, Torres DA, Rojas-Díaz V, Saavedra-Rodríguez CA, Pérez-Torres J. 2017. Mamíferos del departamento de Risaralda, Colombia. Biota Colombiana 18:239-254. https:// doi.org/10.21068/c2017.v18n02a16

Castaño Salazar JH. 2012. Mamíferos de Caldas: Un análisis de vacíos de información. Boletín Científico, Centro de Museos, Museo de Historia Natural 16:101-119.

Castaño Ramírez ND, Ramírez-Chaves HE. 2018. Sistematización y estimación del índice de salud de la Colección de Mamíferos (Mammalia) del Museo de Historia Natural de la Universidad de Caldas, Colombia. Boletín Científico, Centro de Museos, Museo de Historia Natural 22:90-103.

Díaz-Nieto J, Voss RS. 2016. A revision of the didelphid marsupial genus Marmosops, part 1. Species of the subgenus Sciophanes. Bulletin of the American Museum of Natural History 402:1-70. http://dx.doi.org/10.5531/sd.sp.21

Escobar-Lasso S, Cerón-Cardona J, Castaño-Salazar JH. 2013. Los mamíferos de la cuenca del río Chinchiná, en la región andina de Colombia. Therya 4:139-155.

Escobar-Lasso S, Cerón-Cardona J, Castaño-Salazar JH, Mendieta-Giraldo L, Ospina-Herrera O. 2014. Los felinos silvestres del departamento de Caldas, en la región andina de Colombia: composición, distribución y conservación. Therya 5:575-588. http://dx.doi.org/10.12933/therya-14-170

Escobar-Lasso S, Gil-Fernández M. 2014. The highest elevation record of Mustela frenata (Carnivora: Mustelidae) and distribution in Caldas department, Andean region of Colombia. Mammalogy Notes 1:7-9. https:// doi.org/10.47603/manovol1n2.7-9

Escobar-Lasso S, Guzmán-Hernández CF. 2014. El registro de mayor altitud del Hurón Mayor Galictis vittata, con notas sobre su presencia y conservación dentro del departamento de Caldas, en la región andina de Colombia. Therya 5:567-574. http:/ / dx.doi.org/10.12933/therya-14-166

Esquivel DA, Penagos AP, García-R S, Bennett D. 2020. New records of Pygmy Round-eared Bat, Lophostoma brasiliense Peters, 1867 (Chiroptera, Phyllostomidae), and updated distribution in Colombia. Check List 16:277-285. https:// doi.org/10.15560/16.2.277

García Restrepo S, Montilla So, Bustamante Manrique V, Bustamante Manrique S, Cepeda-Duque C, Sánchez Londoño JD, Ramírez-Chaves HE. 2018. Estado de la investigación primatológica en el Eje Cafetero y Antioquia, Colombia. Neotropical Primates 24:56-63.

Marín D, Ramírez-Chaves HE, Suárez-Castro AF. 2012. Revisión cráneo-dentaria de Procyon (Carnivora: Procyonidae) en Colombia y Ecuador, con notas sobre su taxonomía y distribución. Mastozoologia Neotropical 19:259-270.

Marín-C D, Corrales-E JD, Valencia JD. 2018. Nuevos registros de la rata cangrejera Ichthyomys hydrobates (Winge, 1891) (Rodentia: Cricetidae) en Colombia. Mastozoologia Neotropical 25:235-243.

Montilla SO, Cepeda-Duque JC, Bustamante-Manrique S. 2018. Distribución del mono nocturno andino Aotus lemurinus en el departamento del Quindío, Colombia. Mammalogy Notes 4:610. https:// doi.org/10.47603/manovol4n2.6-10 
Morales-Martínez DM, Ramírez-Chaves HE, Colmenares-Pinzón JE, Gómez LG. En prensa. The Koepcke's Spear-nosed bat Gardnerycteris koepckeae (Chiroptera: Phyllostomidae) is not endemic to Peru: First record from the Amazon foothills of Colombia. Mammalia. https://doi.org/10.1515/mammalia-2019-0107

Ramírez-Chaves HE, Mulder KP, Marín D, Pérez WA, Martínez-Arias VM. 2012. Has Colombian Weasel Mustela felipei been overlooked in collections? Small Carnivore Conservation 47:30-34.

Ramírez-Chaves HE, Calderón-Capote MC, Suárez-Castro AF. 2018. The genus Dasyprocta Illiger, 1811 (Mammalia: Rodentia) in Colombia. Mastozoología Neotropical 25:139-149.

Ramírez-Chaves HE, Mejía Fontecha IY, Velásquez D, Castaño D, Ocampo D. 2019. Museo de Historia Natural, Colección de Vertebrados e Invertebrados - Colección de Mamíferos. Version 2.2. Universidad de Caldas. Occurrence dataset accessed via GBIF.org on 2020-03-07. https://doi.org/10.15472/mnevig

Ramírez-Chaves HE, Velásquez-Guarín D, Mejía-Fontecha IY, Ocampo-Velásquez JD, Castaño Ramírez ND. 2020. Colección de mamíferos (Mammalia) del Museo de Historia Natural de la Universidad de Caldas, Colombia. Biota Colombiana 21: in press. https://doi.org/10.21068/c2020.v21n02a11

Roncancio N, Ramírez-Chaves HE. 2008. Registro de leucismo en Sturnira erythromos en los Andes centrales de Colombia. Chiroptera Neotropical 14:412-414.

Roncancio-DN, Estévez-VJ. 2007. Evaluación del ensamblaje de murciélagos en áreas sometidas a regeneración natural y a restauración por medio de plantaciones de aliso. Boletín Científico, Centro de Museos, Museo de Historia Natural 11:131-143.

Sánchez F. 2000. Inventario de mamíferos en un bosque andino del departamento de Caldas, Colombia. Boletín Científico, Centro de Museos, Museo de Historia Natural 4:17-25.

Sánchez F, Alvear M. 2003. Comentarios sobre el uso de hábitat, dieta, y conocimiento popular de los mamíferos en un bosque andino de Caldas, Colombia. Boletín Científico, Centro de Museos, Museo de Historia Natural 7:122-144.

Sánchez F, Sánchez-Palomino P, Cadena A. 2004. Inventario de mamíferos en un bosque de los Andes Centrales de Colombia. Caldasia 26:291-309.

Serna-Botero V, Marín-Martínez M, Velásquez-Guarín D, Ramírez-Chaves HE. 2019. Boyacá spiny rat, Proechimys chrysaeolus (Mammalia: Echimyidae), a new prey item of the banded calico snake, Oxyrhopus petolarius (Reptilia: Dipsadidae). Herpetology Notes 12:651-653.

Torres-Martínez MM., Ramírez-Chaves HE, Noguera-Urbano EA, Colmenares-Pinzón, JE, Passos FC, García J. 2019. On the distribution of the Brazilian porcupine Coendou prehensilis (Erethizontidae) in Colombia. Mammalia 83(3):290-297. https:// doi.org/10.1515/mammalia2018-0043.

Editor: Miguel E. Rodríguez-Posada Recibido: 2020-04-05 Revisado: 2020-04-20 Aceptado: 2020-04-20 Publicado: 2020-05-17 